\title{
Prof. Yin Li: 100-1=0-every step during esophageal surgery should strive for perfection
}

Submitted Nov 25, 2018. Accepted for publication Dec 06, 2018.

doi: $10.21037 /$ jtd.2018.12.29

View this article at: http://dx.doi.org/10.21037/jtd.2018.12.29

\section{Editor's note}

The Third Ruijin International Thoracic Surgery Symposium was successfully held in Shanghai from 15th to 16 th of November in 2018, gathering many renowned experts and scholars from the frontier field at home and abroad to discuss the latest progress and hot issues in thoracic surgery.

During the meeting, we had the privilege of inviting Professor Yin Li (Figure 1), from the Cancer Institute and Hospital, Chinese Academy of Medical Sciences (CAMS), for an interview.

\section{Expert introduction}

Professor Yin Li (Figure 2) is the Director of esophageal Surgery at Cancer Institute and Hospital, CAMS. Before that, he was Vice President of Henan Cancer Hospital, the Affiliated Cancer Hospital of Zhengzhou University in China, as well as the director of the Department of Thoracic Surgery, is an expert on surgical treatment of thoracic cancers such as lung cancer, esophageal cancer, cardia cancer and thymic carcinoma and so forth.

Prof. Li holds numerous academic tittles in China. Internationally, he is also a member of the International Association for the Study of Lung Cancer (IASLC), the International Thymic Malignancy Interest Group (ITMIG) and the European Society of Thoracic Surgeons (ESTS). $\mathrm{He}$ is also the editorial board member of many peerreviewed journals such as Thoracic Cancer and Innovation (Chinese version), to name few.

With all Prof. Li and his team have achieved so far, the biggest accomplishment that makes him known to all is fast-track surgery (FTS) in patients with esophageal cancer, especially the innovative application of "non-tube no fasting" theory. The core of this innovation is to use no nasogastric intubation and encourage early oral feeding postoperatively (at postoperative day 1) in patients following thoracolaparoscopic esophagectomy. This management is

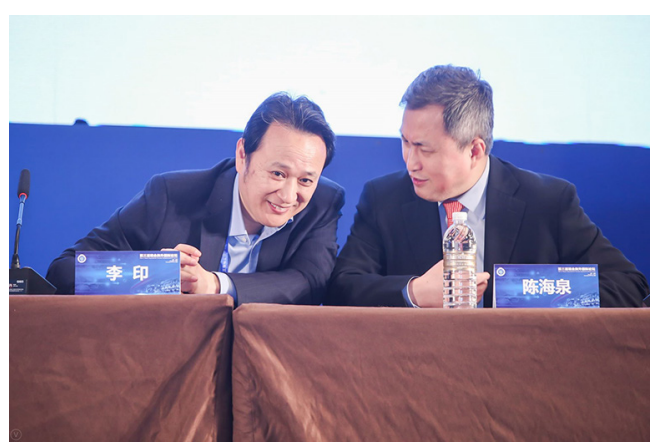

Figure 1 Prof. Yin Li and Prof. Haiquan Chen cochaired a session during the meeting.

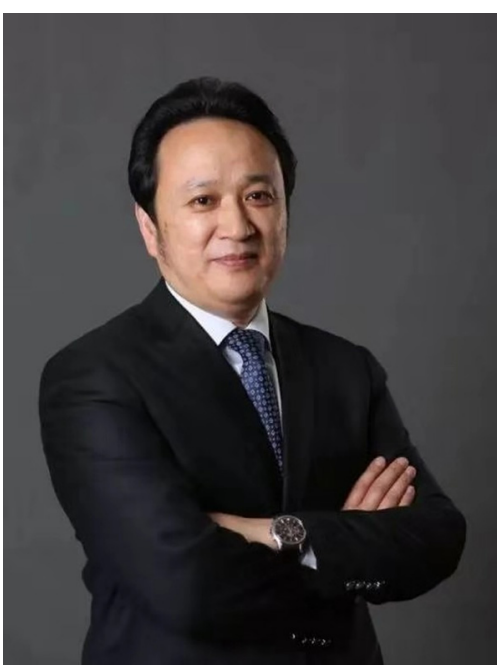

Figure 2 Prof. Yin Li.

proven to be feasible and safe clinically. To gather more scientific evidence, a phrase III clinical trial directed by Prof. $\mathrm{Li}$ is underway, with an interim analysis published as poster presentation during American Association for Thoracic Surgery (AATS) 2015.

In addition, the Li's Anastomosis, a creative surgical method developed by Prof. Yin Li based on many 
traditional ways against anastomotic leak, tremendously improves the surgical outcome and plays a crucial role in FTS for esophageal cancer patients.

\section{Interview questions}

(I) ERAS Multi-Center Clinical Research Group on "Non-Tube No Fasting" Fast Track Program for Resectable Esophageal Carcinoma was started last year. Would you like to briefly introduce current focus of the group?

(II) Would you like to talk about the necessity of preventing complications during perioperative period of esophageal surgery?

(III) What do you think is most important progress made in the concept and practice of ERAS thoracic surgery in China over the past years?

(IV) Do you think there are room in perfecting the "nontube no fasting" model and management of FTS in patients with esophageal cancer?

(V) At present, what are the new trends in the basic and clinical fields of esophageal cancer that deserve special attention?

\section{Interview summary}

At the beginning, Professor Yin Li briefly introduced the progress of the Enhanced Recovery After Surgery (ERAS) Multi-Center Clinical Research Group on "Non-Tube No Fasting" Fast Track Program for Resectable Esophageal Carcinoma. He said that currently he and his team is focusing on well-designed training program for surgeons. He believes that through the systematic training, surgeons in this field can develop a technical consensus in clinical practice, which can better pave the path for the incoming clinical research work.

With regard to the significance of preventing complications during perioperative period of esophageal surgery, Prof. Li empathized that the prevention is a systemic work-every step during the procedure matters. If there is one thing that fails to be noticed, even very small one, it may be $100-1=0$, a trip in one point would have spoiled all. As a result, patients will end up suffering very serious and even fatal complications. Therefore, from the beginning to the end, every step of the operation should strive for perfection. In addition, attention needs to be paid to the protection of the related tissues and organs as well. In summary, if all the surgical operations can be completed with high quality, the procedure will be definitely a success.

When we asked about the most important progress made in the concept and practice of ERAS thoracic surgery in China over the past years, Prof. Li believes that we have made significant breakthroughs in the following aspects. First of all, "non-tube no fasting" is an innovative management of FTS in patients with esophageal cancer, making it possible for patients resuming euphagia shortly. Usually, patients undergoing traditional esophageal cancer treatment will be required quite a long time in fasting, which is detrimental for their rapid recovery and places a great burden on their economy as well. Prof. Li hence is proud that realizing early eating is the biggest change in the treatment mode of esophageal cancer, which has a landmark significance in the field of esophageal surgery. Another breakthrough is the establishment of a gastric protection system and method for the treatment of esophageal cancer, which Prof. Li believes should popularize.

FTS in patients with esophageal cancer is the biggest accomplishment that makes him known to all, especially the innovative application of "non-tube no fasting" theory. When asked whether there are room for perfecting, "till good is better, but better best." Prof. Li replied. He explained that all surgical techniques and treatment models can only be perfected through continuous practice, the renewal of new drugs, the constant updating of new instruments and equipment.

He had suggested ten years ago that to solve problems of patient's normal eating, diarrhea, cough and the protection of the vagus nerve and stomach during surgery are pressing tasks in treating esophageal cancer. Currently, the problem of normal eating has been basically solved by "non-tube no fasting" approach. However, the protection of the vagus nerve and stomach during surgery still presents a challenge for surgeons. Esophageal cancer surgery cost surgeons plenty of time and energy. Thus, in order to better protect vagus nerve and the stomach, the finer operations are required, which Prof. Li thought the system of "on-tube no fasting" should consummate for.

Prof. Li also pointed out that China has not yet had seamless connection between in-home care, community health service and the hospitals. He hopes that a system bridging these three can be well established in the near future.

In the end of the interview, Prof. Li said from the experiences fighting against lung cancer, breast cancer and 
colorectal cancer, we can see that the discovery of driving genes, related targets and the development of drugs have greatly contributed to advancing the treating progress. But basic research on esophageal cancer is much inferior, so it is more necessary to redouble efforts to make results in targeted therapy, drive genes, and immunotherapy. Moreover, Prof. Li said that it is very important to combine clinical research and basic research and find out the dominant people, together to work out better treatment for patients.

Cite this article as: Yuan A. Prof. Yin Li: 100-1=0_every step during esophageal surgery should strive for perfection. J Thorac Dis 2018;10(12):E857-E859. doi: 10.21037/jtd.2018.12.29

\section{Acknowledgements}

The author would like to thank Prof. Yin Li for participating in the interview.

\section{Footnote}

Conflicts of Interest: The author has no conflicts of interest to declare.

(Science Editor: Amber Yuan, JTD, jtd@amepc.org) 